\title{
Exserohilum Infections Associated with Contaminated Steroid Injections
}

\section{A Clinicopathologic Review of 40 Cases}

Jana M. Ritter, ${ }^{*}$ Atis Muehlenbachs, ${ }^{*}$ Dianna M. Blau, ${ }^{*}$ Christopher D. Paddock, ${ }^{*}$ Wun-Ju Shieh, ${ }^{*}$ Clifton P. Drew, ${ }^{*}$ Brigid C. Batten, ${ }^{*}$ Jeanine H. Bartlett, ${ }^{*}$ Maureen G. Metcalfe, ${ }^{*}$ Cau D. Pham ${ }^{\dagger}$ Shawn R. Lockhart, ${ }^{\dagger}$ Mitesh Patel, ${ }^{*}$ Lindy Liu, ${ }^{*}$ Tara L. Jones, ${ }^{*}$ Patricia W. Greer, ${ }^{*}$ Jeltley L. Montague, ${ }^{*}$ Elizabeth White, ${ }^{*}$ Dominique C. Rollin, ${ }^{*}$ Cynthia Seales, Donna Stewart, ${ }^{\ddagger}$ Mark V. Deming, ${ }^{\S}$ Mary E. Brandt, ${ }^{\dagger}$ Sherif R. Zaki*; for the Exserohilum Infections Working Group

From the Infectious Diseases Pathology Branch,* Division of High Consequence Pathogens and Pathology, and the Mycotic Diseases Branch, ${ }^{\dagger}$ Division of Foodborne, Waterborne, and Environmental Diseases, Centers for Disease Control and Prevention, Atlanta, Georgia; the Office of the Chief Medical

Examiner ${ }^{\ddagger}$ Louisville, Kentucky; and the Department of Pathology, ${ }^{\S}$ University of Michigan, Ann Arbor, Michigan

CME Accreditation Statement: This activity ("ASIP 2013 AJP CME Program in Pathogenesis") has been planned and implemented in accordance with the Essential Areas and policies of the Accreditation Council for Continuing Medical Education (ACCME) through the joint sponsorship of the American Society for Clinical Pathology (ASCP) and the American Society for Investigative Pathology (ASIP). ASCP is accredited by the ACCME to provide continuing medical education for physicians.

The ASCP designates this journal-based CME activity (“ASIP 2013 AJP CME Program in Pathogenesis”) for a maximum of 48 AMA PRA Category 1 Credit(s) ${ }^{\top M}$. Physicians should only claim credit commensurate with the extent of their participation in the activity.

CME Disclosures: The authors of this article and the planning committee members and staff have no relevant financial relationships with commercial interests to disclose.

Accepted for publication

May 20, 2013.

Address correspondence to Jana M. Ritter, D.V.M., Infectious Diseases Pathology Branch, Centers for Disease Control and Prevention, Mailstop G-32, 1600 Clifton Rd NE, Atlanta, GA 30333. E-mail: jritter@cdc. gov.

\begin{abstract}
September 2012 marked the beginning of the largest reported outbreak of infections associated with epidural and intra-articular injections. Contamination of methylprednisolone acetate with the black mold, Exserohilum rostratum, was the primary cause of the outbreak, with $>13,000$ persons exposed to the potentially contaminated drug, 741 confirmed drug-related infections, and 55 deaths. Fatal meningitis and localized epidural, paraspinal, and peripheral joint infections occurred. Tissues from 40 laboratoryconfirmed cases representing these various clinical entities were evaluated by histopathological analysis, special stains, and IHC to characterize the pathological features and investigate the pathogenesis of infection, and to evaluate methods for detection of Exserohilum in formalin-fixed, paraffin-embedded (FFPE) tissues. Fatal cases had necrosuppurative to granulomatous meningitis and vasculitis, with thrombi and abundant angioinvasive fungi, with extensive involvement of the basilar arterial circulation of the brain. IHC was a highly sensitive method for detection of fungus in FFPE tissues, demonstrating both hyphal forms and granular fungal antigens, and PCR identified Exserohilum in FFPE and fresh tissues. Our findings suggest a pathogenesis for meningitis involving fungal penetration into the cerebrospinal fluid at the injection site, with transport through cerebrospinal fluid to the basal cisterns and subsequent invasion of the basilar arteries. Further studies are needed to characterize Exserohilum and investigate the potential effects of underlying host factors and steroid administration on the pathogenesis of infection. (Am J Pathol 2013, 183: 881-892; http://dx.doi.org/10.1016/j.ajpath.2013.05.007)
\end{abstract}

The findings and conclusions herein are those of the authors and do not necessarily represent the official position of the Centers for Disease Control and Prevention.

Exserohilum Infections Working Group: John A. Jernigan, J. Todd Weber, Melissa Schaefer, Tom M. Chiller, Monika Roy, Benjamin J. Park, Angela A. Cleveland, Anastasia P. Litvintseva, Rachel M. Smith, Anne
Purfield, Marlene DeLeon-Carnes, Amy M. Denison, Julu Bhatnagar, Cynthia S. Goldsmith, and Jevon McFadden (CDC); Marion A. Kainer (Tennessee Department of Health); Denise A. Pettit (Virginia Division of Consolidated Laboratory Services); Carla M. Davis (St. Thomas Hospital, Nashville, TN); and Wendy Lavezzi (Florida District 5 Medical Examiner's Office). 
Epidural and intra-articular steroid injections are commonly administered for treatment of back, neck, and peripheral joint pain, especially in elderly patients. Infections develop in an estimated $<1 \%$ to $2 \%$ of patients who receive spinal injections. ${ }^{1-3}$ Introduction of skin flora into the injection site is the most common cause of infection, and Staphylococcus aureus is the most commonly implicated organism. ${ }^{2,4-6}$ Fungal infections associated with spinal injections are exceedingly rare and are most frequently attributed to Aspergillus. ${ }^{7-9}$ Although individual cases of infection are uncommon, outbreaks of infection associated with contaminated epidural or intra-articular injections are even more unusual. During the past decade, such examples include three outbreaks of Serratia marcescens infection associated with epidural or intra-articular injections, ${ }^{10-12}$ an outbreak of Klebsiella pneumoniae and Enterobacter aerogenes bacteremia after sacroiliac (SI) joint steroid injections, ${ }^{13}$ an outbreak of Exophiala dermatitidis infection after epidural and intraarticular steroid injections, ${ }^{14}$ and an outbreak of Aspergillus meningitis after spinal anesthesia for caesarean section. ${ }^{7}$ The largest of these outbreaks involved 11 patients. ${ }^{10}$

In September 2012, the Centers for Disease Control and Prevention (CDC) was alerted to multiple reports of fungal meningitis in patients who had recently received epidural steroid injections. The intensive, multistate, epidemiological and laboratory investigation that ensued identified an unprecedented outbreak of fungal infections associated with epidural, paraspinal, and peripheral joint injections, involving three contaminated lots of preservative-free methylprednisolone acetate (MPA) from a single compounding pharmacy. More than 17,000 vials from three contaminated lots were distributed to medical centers across the United States, and $>13,000$ people were injected with the potentially contaminated drug before discovery of the source of contamination. ${ }^{15}$ Through May 6, 2013, 741 cases and 55 deaths in 20 states have been associated with this outbreak, making it the largest recognized cluster of infections associated with epidural and intra-articular steroid injections. Most cases with a confirmed etiology have been attributed to Exserohilum rostratum. In few cases, other fungal or bacterial species have been identified by molecular studies or culture, either individually or in addition to Exserohilum. ${ }^{16,17}$ Herein, we describe the pathological findings from 40 case patients from this outbreak with confirmed Exserohilum infection, correlate these findings with clinical and laboratory data, and use these data to provide insight into the pathogenesis of Exserohilum infections after injection of contaminated MPA.

\section{Materials and Methods}

\section{Case Selection}

Tissues from 40 case patients, for whom Exserohilum was identified, were sequentially selected in this case series. Patient classifications were adapted from the CDC's clinical case definitions for the fungal meningitis outbreak (http://www.cdc.gov/hai/outbreaks/clinicians/index.html\#case, last accessed January 1, 2013) and were assigned based on patients' clinical histories, as reported by their treating clinicians. Case patients were classified as persons who received a preservative-free MPA injection from one of the contaminated lots, subsequently developed any of the following conditions, and had laboratory confirmation of Exserohilum infection: A) meningitis of unknown etiology after epidural or paraspinal injection, B) abscess or other infection of unknown etiology in the spinal or paraspinal structures at or near the site of injection after epidural or paraspinal injection, and C) worsening inflammatory arthritis of a peripheral joint of unknown etiology diagnosed after joint injection. One of the patients included in this series has been previously detailed in a case report. ${ }^{18}$

Clinical samples, including cerebrospinal fluid (CSF), joint fluid, and tissues obtained at biopsy or autopsy from case patients, were submitted to the CDC for laboratory evaluation. Tissues were examined microscopically and tested by special stains and immunohistochemistry (IHC) for evidence of fungal or other infectious agents. Fresh or paraffin-embedded tissue samples were also evaluated by PCR analyses.

\section{Clinical and Epidemiological Data}

Completeness of accompanying clinical and demographic information varied considerably. The following data, when available, were recorded for each patient: age, sex, case definition, location of injection, date of most recent MPA injection, date of initiation of antifungal treatment, date of tissue collection, date of death (if applicable), and any pertinent gross autopsy or surgical findings.

\section{Histopathology}

Fresh, frozen, formalin-fixed, or paraffin-embedded tissues were received for evaluation. Tissues were fixed in $10 \%$ neutral-buffered formalin and processed for routine histological analysis, sectioned, and stained with $H \& E$ and Grocott's methenamine silver (GMS). In select cases, tissues were also stained with PAS or Fontana-Masson (FM) techniques. Tissue sections were evaluated for inflammation (scored categorically as necrosuppurative, granulomatous, lymphohistiocytic, with or without necrosis/degeneration, or unremarkable) and for amount of fungus seen (fungal burden, scored from 0 to 3 , where 0 indicates none; 1 , few; 2, moderate; and 3, abundant).

\section{IHC Assays}

IHC assays were performed using a polymer-based indirect immunoalkaline phosphatase detection system with colorimetric detection of antibody/polymer complex with Fast Red Chromogen (Thermo Fisher Scientific, Runcom, Cheshire, UK, or Biocare Medical, Concord, CA). Tissues were evaluated with a polyfungal antibody composed of a pool of four 
polyclonal antibodies, generated to several different fungi, and shown in our laboratory to cross-react with many different fungal species, including Exserohilum (Table 1). Polyfungal IHC is not specific for Exserohilum but detects a wide range of fungal species; therefore, cases that were positive by polyfungal IHC and lacked other laboratory confirmation of Exserohilum were also tested by IHC using more specific monoclonal anti-Aspergillus species and monoclonal anti-zygomycete antibodies to exclude infection with these fungi. Appropriate positive and negative control serum and tissues were tested in parallel for each assay.

\section{Electron Microscopy}

Formalin-fixed tissues or formalin-fixed, paraffin-embedded (FFPE) sections from four cases were processed for transmission electron microscopy. Tissues were rinsed with a sodium phosphate buffer ( $\mathrm{pH} 7.3$ ) before fixing in $2.5 \%$ glutaraldehyde, and FFPE sections were deparaffinized and rehydrated as previously described. ${ }^{19}$ Specimens were then post-fixed in osmium tetroxide and enblock stained with $4 \%$ aqueous uranyl acetate. Microwave irradiation (Ted Pella Biowave; Ted Pella, Inc., Redding, CA) was used to facilitate ethanol dehydration and acetone and resin infiltration [Epon substitute and Araldite (Ted Pella, Inc.)]. ${ }^{20}$ Resin-embedded specimens were polymerized overnight in an oven at $60^{\circ} \mathrm{C}$.

\section{Molecular Detection of Fungus in Tissues}

Molecular identification of fungi in fresh or FFPE tissue samples was achieved by PCR and DNA sequence analysis of the fungal internal transcribed spacer region, as described by Gade et al. ${ }^{21}$ Total nucleic acid was purified from fresh tissues by using the QIAamp DNeasy Blood and Tissue Kit (Qiagen, Gathersburg, MD), according to the manufacturer's instructions, with minor modifications. ${ }^{21}$ For FFPE tissue, 5 to 10 scrolls of sections ( $5 \mu \mathrm{m}$ thin) were used for DNA extraction. ${ }^{22}$

\section{Statistical Analysis}

For all statistical tests, significance was defined as $P<0.05$. Data analysis was performed using SAS software version 9.3 (SAS Institute Inc., Cary, NC). Bivariate analysis was performed by $\chi^{2}$ or Fisher's exact test. A multivariate logistic regression model was used to examine the time interval between injection and tissue collection.

\section{Results}

\section{Clinical and Epidemiological Data}

Tissues were received between October 11, 2012, and January 24, 2013, from case-patients from seven US states (Florida, Indiana, Maryland, Michigan, North Carolina, Tennessee, and Virginia). Of the patients, $65 \%$ were women. Ages ranged from 34 to 92 years (median, 67 years); $93 \%$ of patients were 50 years or older (Table 2 ). The interval between injection and tissue collection, when reported $(n=38)$, ranged from 12 to 120 days (median, 52 days). Of 34 patients whose treatment status was reported, $31(91 \%)$ received antifungal therapy. Of 40 cases, $16(40 \%)$ were fatal; all but two fatal cases had a clinical diagnosis of meningitis.

\section{Specimen Classification}

The anatomical site of injection was reported for $36(90 \%)$ of 40 case patients and included lumbar spine $(n=22)$, cervicothoracic spine $(n=5)$, SI joint $(n=5)$, and hip joint $(n=4)$ (Table 2). Eleven patients had a reported clinical history of only meningitis, 6 had meningitis and epidural or paraspinal injection site infection, 19 had injection site infection only, and four had peripheral joint infection. Of 11 patients with reported meningitis, central nervous system (CNS) tissues (brain and spinal cord other than injection site) were received for all patients; injection site tissues were also received for 6 of the 11 patients, despite lack of clinical evidence of injection site infection, to investigate for persistence of fungus and inflammation at the injection site. Of six patients with meningitis and injection site infection, CNS and injection site tissues were received for only three. For the other three patients, only injection site tissues were received. Of 19 patients with only epidural/paraspinal infection, 14 involved infection of the epidural injection site, whereas five specifically involved the SI joint. For these cases, only tissues from the area of the injection site were received. Biopsy tissues were evaluated from four patients with peripheral joint (hip) infection.

Table 1 Primary Antibodies Used for IHC

\begin{tabular}{|c|c|c|c|c|}
\hline Antibody name & Confirmed cross-reactivity & Serum source & Dilution & Source \\
\hline Polyfungal pool (pAb) & $\begin{array}{l}\text { Blastomyces, Histoplasma, Coccidioides, Candida, } \\
\text { Cryptococcus, Aspergillus, Basidiobolus, Fusarium } \\
\text { oxysporum, Apophyomyces, Microsphaeropsis, } \\
\text { and Exserohilum }\end{array}$ & Goat & $1: 2000$ & $\begin{array}{l}\text { Meridian } \\
\quad \text { Diagnostics } \\
\quad \text { (Cincinnati, } \mathrm{OH})\end{array}$ \\
\hline Aspergillus species (mAb) & Aspergillus, Histoplasma, and Penicillium & Mouse & $1: 1000$ & $\begin{array}{l}\text { Dako Corp } \\
\quad(\text { Carpinteria, CA) }\end{array}$ \\
\hline
\end{tabular}

$\mathrm{mAb}$, monoclonal antibody; pAb, polyclonal antibody. 
Table 2 Clinicoepidemiologic Data and Histopathologic Findings

\begin{tabular}{|c|c|c|c|c|c|c|c|}
\hline Variable & \multicolumn{2}{|l|}{ Meningitis } & \multicolumn{2}{|c|}{$\begin{array}{l}\text { Meningitis + IS } \\
\text { infection }\end{array}$} & $\begin{array}{l}\text { Epidural/ } \\
\text { paraspinal IS } \\
\text { infection only }\end{array}$ & $\begin{array}{l}\text { Peripheral joint } \\
\text { infection }\end{array}$ & $\begin{array}{l}\text { All case } \\
\text { patients }\end{array}$ \\
\hline No. of case patients & \multicolumn{2}{|l|}{11} & \multicolumn{2}{|l|}{6} & 19 & 4 & \\
\hline \multicolumn{8}{|l|}{ Patient demographics } \\
\hline No. $(\%)$ received antifungal treatment & \multicolumn{2}{|l|}{$8(73)$} & \multicolumn{2}{|l|}{$6(100)$} & $14(74)$ & $3(75)$ & $31(78)$ \\
\hline No. $(\%)$ not received antifungal treatment & \multicolumn{2}{|l|}{$3(27)$} & \multicolumn{2}{|l|}{$0(0)$} & $0(0)$ & $0(0)$ & $3(8)$ \\
\hline No. (\%) with treatment status unknown & \multicolumn{2}{|l|}{$0(0)$} & \multicolumn{2}{|l|}{$0(0)$} & $5(26)$ & $1(25)$ & $6(15)$ \\
\hline No. (\%) fatal & \multicolumn{2}{|l|}{$11(100)$} & \multicolumn{2}{|l|}{$3(50)$} & $2(11)$ & $0(0)$ & $16(40)$ \\
\hline Interval range (median) injection to tissue & \multirow{2}{*}{\multicolumn{2}{|c|}{$12-79(38)[n=11]$}} & \multirow{2}{*}{\multicolumn{2}{|c|}{$22-81(56)[n=6]$}} & $36-111(64)$ & $50-120(66)$ & $12-120(52)$ \\
\hline collection (days) & & & & & {$[n=17]$} & {$[n=4]$} & {$[n=38]$} \\
\hline Cervicothoracic spine & \multicolumn{2}{|l|}{3} & \multicolumn{2}{|l|}{0} & 2 & & $5(12)$ \\
\hline Sacroiliac joint & & & & & 5 & & $5(12)$ \\
\hline Hip joint & & & & & & 4 & $4(10)$ \\
\hline Not specified & 0 & & 1 & & 3 & 0 & $4(10)$ \\
\hline $\begin{array}{l}\text { Predominant histopathological } \\
\text { characteristics }\end{array}$ & Brain & IS & Brain & IS & IS & IS & \\
\hline Necrosuppurative inflammation & $5^{*}$ & 0 & 0 & 1 & 3 & 1 & \\
\hline $\begin{array}{l}\text { Necrosuppurative and granulomatous } \\
\text { inflammation }\end{array}$ & $2^{*}$ & 3 & $1^{*}$ & 2 & 1 & 2 & \\
\hline Lymphocytic vasculitis & 1 & 0 & 0 & 0 & 0 & 0 & \\
\hline
\end{tabular}

*With vasculitis, thrombosis, and fungal angioinvasion.

IS, injection site.

\section{Gross Pathology}

The most frequently reported gross autopsy findings for cases of fatal meningitis were diffuse meningeal congestion and hemorrhage and apparent necrosis involving one or more areas of the brain and/or spinal cord (cortex, thalamus, basal ganglia, cerebellum, brainstem, or upper spinal cord). Extensive hemorrhage around the base of the brain and thrombi involving the basilar arterial circulation were also commonly reported (Figure 1, A and B).

\section{Histopathological Findings}

\section{Meningitis}

Of case patients for whom brain tissue was submitted for evaluation of meningitis, the most consistent finding was acute to subacute necrosuppurative meningitis with vasculitis, thrombosis, and angioinvasive fungi (Figure 1, C-H, and Table 2). The leptomeninges covering the basilar surfaces of the midbrain and brainstem, and the associated large arterial supplies to the brain (circle of Willis and basilar, vertebral, cerebellar, and middle cerebral arteries), were most frequently and extensively involved. Fibrinosuppurative exudates were centered on and around arteries, with greatest involvement of the necrotic adventitial surface and progressive transmural involvement to the lumen in more severely affected vessels, which were often thrombosed (Figure 1, C-E). Numerous fungal elements were present within the perivascular exudate and within arterial walls, and to a lesser extent within vascular lumens (Figure 1, F and G). In several cases, fungi unaccompanied by inflammation were present within the tunica media of intact arterial segments (Figure 1H). A subset of cases had prominent granulomatous infiltrates in addition to necrosuppurative inflammation. In these cases, fungal elements were abundant, and phagocytosed fungal fragments were consistently identified within multinucleated giant cells (Figure 2, A and B). Giant cells surrounded and infiltrated arterial walls, in one case resulting in pronounced dissection of 

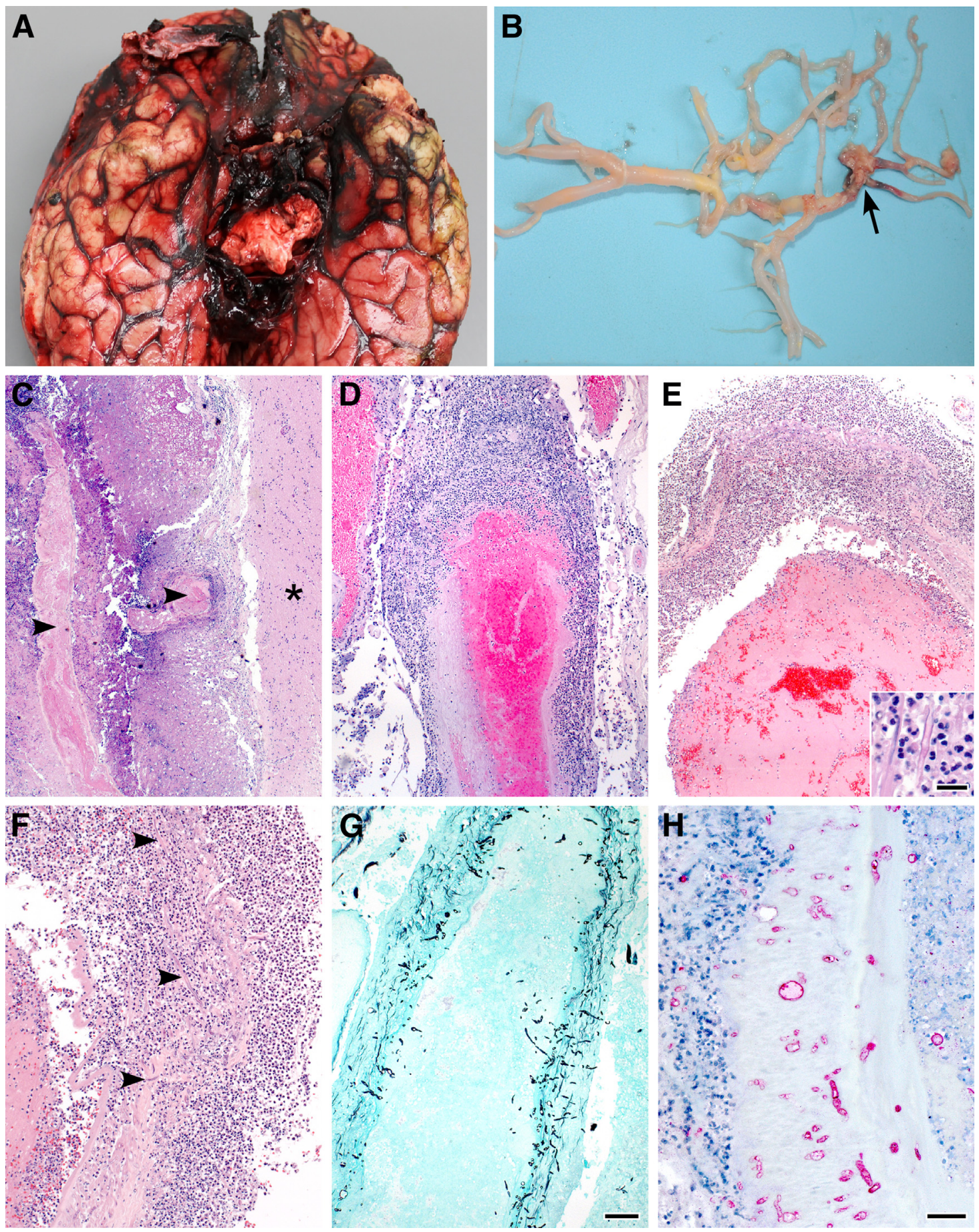

Figure 1 Most frequent gross and histopathological features of acute fatal Exserohilum meningitis. A: Hemorrhage around base of brain. B: Cerebral artery thrombus (arrow), as part of the circle of Willis. C: Expansion of meninges by necrosuppurative infiltrate and vascular thrombosis (arrowheads). The asterisk indicates brain parenchyma. D: Infiltration of a large meningeal arterial wall by neutrophils. E: Infiltration and destruction of the middle cerebral arterial wall by neutrophils (inset) and a large luminal thrombus. F: Fungal elements (arrowheads) admixed with the mural inflammatory infiltrate, higher magnification of same section as E. G: GMS stain highlights numerous fungal hyphae within a large arterial wall. $\mathbf{H}$ : Polyfungal IHC shows many fungi unaccompanied by inflammation in the tunica media of a large cerebral artery. H\&E staining (C-F); GMS staining (G); polyfungal immunoalkaline phosphatase staining, naphthol fast red substrate with hematoxylin counterstain (H). Original magnification: $\times 6.75(\mathbf{C}) ; \times 12.5(\mathbf{D}$ and $\mathbf{G}) ; \times 12.5(\mathbf{E}) ; \times 158(\mathbf{E}$, inset); $\times 25(\mathbf{F}) ; \times 50(\mathbf{H})$. Scale bars: $10 \mu \mathrm{m}(\mathbf{E}) ; 100 \mu \mathrm{m}(\mathbf{G}) ; 40 \mu \mathrm{m}(\mathbf{H})$.

the tunica media with aneurysmal dilation (Figure 2C). In one case, brain and spinal meninges showed predominately marked lymphocytic vasculitis and perivasculitis of the middle cerebral artery and arteries of the posterior circle of
Willis, with massive meningeal hemorrhage but without the fibrinosuppurative or granulomatous exudates characteristic of most cases. Three cases had only patchy lymphohistiocytic perivascular meningeal infiltrates in examined sections. 

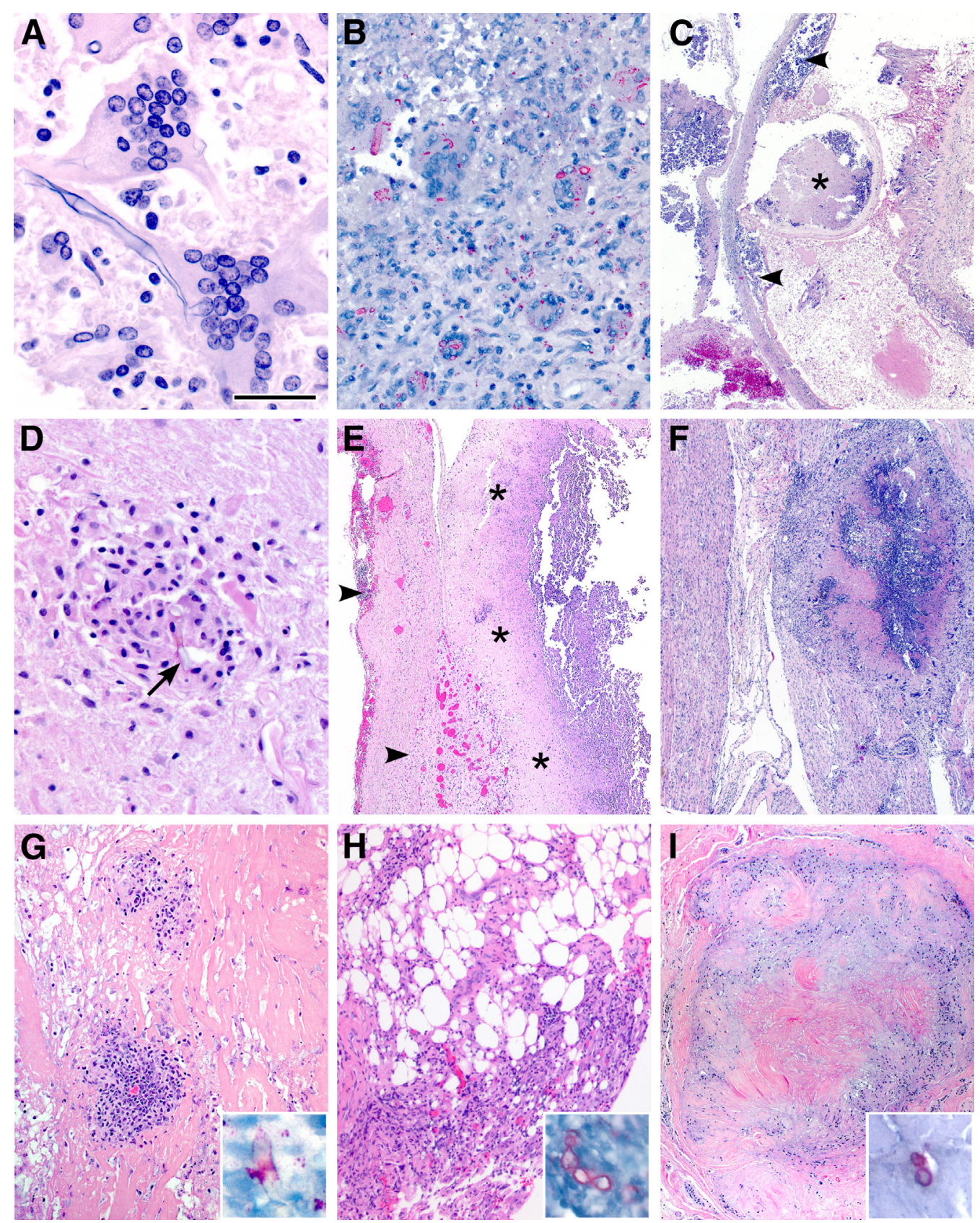

Figure 2 Additional features of Exserohilum meningitis (A-D) and features of injection site pathology (E-I). A: Multinucleated giant cells with intracytoplasmic fungal elements in a patient with granulomatous meningitis. B: Polyfungal IHC shows abundant fungal fragments within giant cells. C: Dissection of a basilar arterial wall by granulomatous infiltrates (arrowheads), with aneurysm formation (asterisk) as the result of accumulation of inflammatory debris within the tunica media. D: A single fungal fragment (arrow) within a granuloma in the deep pontine parenchyma. E: Disruption of the dura by chronic inflammation and neovascularization (arrowheads); necrosuppurative exudate subjacent to the dura (asterisks). F: Necrotizing granulomatous cauda equina neuritis associated with lumbar epidural steroid injection. G: Paraspinal collagenous tissue with necrosis and multiple foci of inflammation. Inset: A fragment of a fungal hypha within a focus of inflammation (Polyfungal IHC). H: Epidural adipose tissue with lymphohistiocytic infiltrates. Inset: Chain of fungal hyphae amid the inflammation (Polyfungal IHC). I: Necrotizing granuloma within a hip joint synovial biopsy specimen. Inset: Fungal hyphae in the central area of necrosis (Polyfungal IHC). H\&E staining (A and $\mathbf{C}-\mathbf{I})$; polyfungal immunoalkaline phosphatase staining, naphthol fast red substrate with hematoxylin counterstain (B and insets of G-I). Original magnification: $\times 158(\mathbf{A}) ; \times 50(\mathbf{B}$ and $\mathbf{D}) ; \times 6.75(\mathbf{C}$ and $\mathbf{F})$; $\times 12.5(\mathbf{E}$ and $\mathbf{I}) ; \times 25(\mathbf{G}$ and $\mathbf{H})$. Scale bar $=30 \mu \mathrm{m}(\mathbf{A})$.

Fungal invasion of brain parenchyma was notably infrequent. CNS tissues of only one patient had evidence of fungus in the deep brain parenchyma. In this case, the pontine parenchyma deep to an area of meningeal inflammation showed a small nodular focus of granulomatous inflammation containing a single fungal fragment consistent with a thromboembolic fungal granuloma (Figure 2D). There was no evidence of direct spread of 
infection from the meninges into the subjacent parenchyma in brain or spinal cord away from the injection site in any patient. In two patients, there was multifocal intraparenchymal small-vessel vasculitis and thrombosis with acute infarction of the surrounding parenchyma. One case had evidence of chronic infarcts; no fungal hyphae were identified in these areas.

\section{Injection Sites: Epidural/Paraspinal Soft Tissue and Spinal Cord}

For case patients with fatal meningitis for whom tissues were submitted from the epidural injection site, histopathological features were similar to those seen in the brain and included necrosuppurative to granulomatous dural, epidural, and leptomeningeal infiltrates with fungus frequently identified (Figure $2 \mathrm{E}$ and Table 2). When spinal cord and/or cauda equina near the injection site was submitted for pathological evaluation, inflammation and fungi often infiltrated spinal nerve roots and nerves of the cauda equina (Figure 2F).

Of patients for whom only epidural or paraspinal infections at the site of injection (including SI joint injections) were reported, submitted tissues comprised fragments of various connective tissues, including epidural fibroadipose tissue and skeletal muscle, ligament, dura, intervertebral disk, and vertebral bone. Tissues from only 4 (21\%) of 19 patients had prominent necrosuppurative inflammation with fungi readily identified by $\mathrm{H} \& \mathrm{E}$ stain. Of 19 patients, 14 (74\%) had lymphohistiocytic inflammation with or without foci of necrosis; in these cases, fungi were only infrequently [2 (14\%) of 14] identified by H\&E, but were detected by IHC in all cases (Figure 2, G and $\mathrm{H}$ ).

\section{Peripheral (Hip) Joints}

Hip joint synovial biopsy specimens were evaluated for four patients. All had necrosis and suppurative, granulomatous, or lymphohistiocytic inflammation (Table 2). Fungus was seen by H\&E in only one case, but was detected by IHC in all cases (Figure 2I).

\section{Extra-CNS Tissues}

For seven patients with fatal meningitis, multiple extra-CNS tissues, including heart, lung, liver, and kidney, were also submitted for histopathological evaluation. Although four cases had evidence of bacterial bronchopneumonia $[S$. aureus $(n=2)$, Prevotella species $(n=1)$, and Streptococcus species $(n=1)$ ], no tissues for any case had histopathological evidence of vasculitis or inflammation similar to that observed in the brain, and fungus was not identified by $\mathrm{H} \& \mathrm{E}$ or IHC in these tissues.

\section{Fungal Morphology}

In H\&E-stained tissue sections, fungal elements appeared as basophilic to pale brown hyphae of variable width (5 to 10 $\mu \mathrm{m})$ with bulbous dilations and occasional septation and nondichotomous branching. Less commonly, chains of yeast-like structures, elongated septate conidia, and individual large (diameter, up to $30 \mu \mathrm{m}$ ) spherical chlamydospore-like forms were also seen (Figure 3, A-D). PAS and GMS stains identified most Exserohilum forms (Figure 3E). Fontana-Masson also detected Exserohilum hyphae with similar sensitivity as PAS and GMS but was considered easier to interpret due to less background nonspecific staining (Figure 3F).

\section{Immunohistochemistry}

Polyfungal IHC was a highly sensitive method for detection of fungus, with antigen detected in all cases, compared with fungi seen in $17(43 \%)$ and $38(95 \%)$ of 40 cases with H\&E and GMS stains, respectively (Table 3). In general, there was more widespread staining with IHC than with GMS stain. Both fungal hyphae and granular fungal antigen were detected by IHC, but conidia were not readily labeled. Hyphae were usually abundant in areas of necrosuppurative inflammation (Figure 3G), but were also seen in noninflamed connective tissues, including ligament and cartilage (Figure 3H); hyphae were not detected in bone or bone marrow spaces. IHC detection of granular antigens within foci of necrosis or lymphohistiocytic infiltrates was particularly useful for diagnosing infection at epidural/paraspinal injection sites, where distinct fungal elements were often rare (Figure 3I). Tissues that were IHC positive by polyfungal IHC and had no other laboratory confirmation of Exserohilum were also tested by IHC to exclude other fungi; no such case included in this series was positive by IHC for Aspergillus species or a zygomycete fungus.

\section{Electron Microscopy}

Electron microscopic evaluation of affected arteries revealed infiltration of vessel walls by fungal hyphae, which were seen both between and within smooth muscle cells (Figure 3J). As appreciated on H\&E, there was often minimal to no inflammatory response surrounding intramural hyphae. In the adventitia, hyphae were present within and parallel to bundles of dense collagen fibers. There was apparent dissolution of surrounding collagen fibrils, with replacement by electron-dense material, suggesting digestion/hydrolysis by the hyphae (Figure 3K).

\section{Correlation of Histopathology with Clinical and Epidemiological Data}

In this series, cases with meningitis only-all of which were fatal-had a shorter interval between injection and tissue collection compared with all other cases $(P=0.009)$. Among all meningitis cases (with or without concurrent injection site infection), tissues with significant pathological features (necrosuppurative, with or without granulomatous inflammation) were more likely to have a high fungal burden 

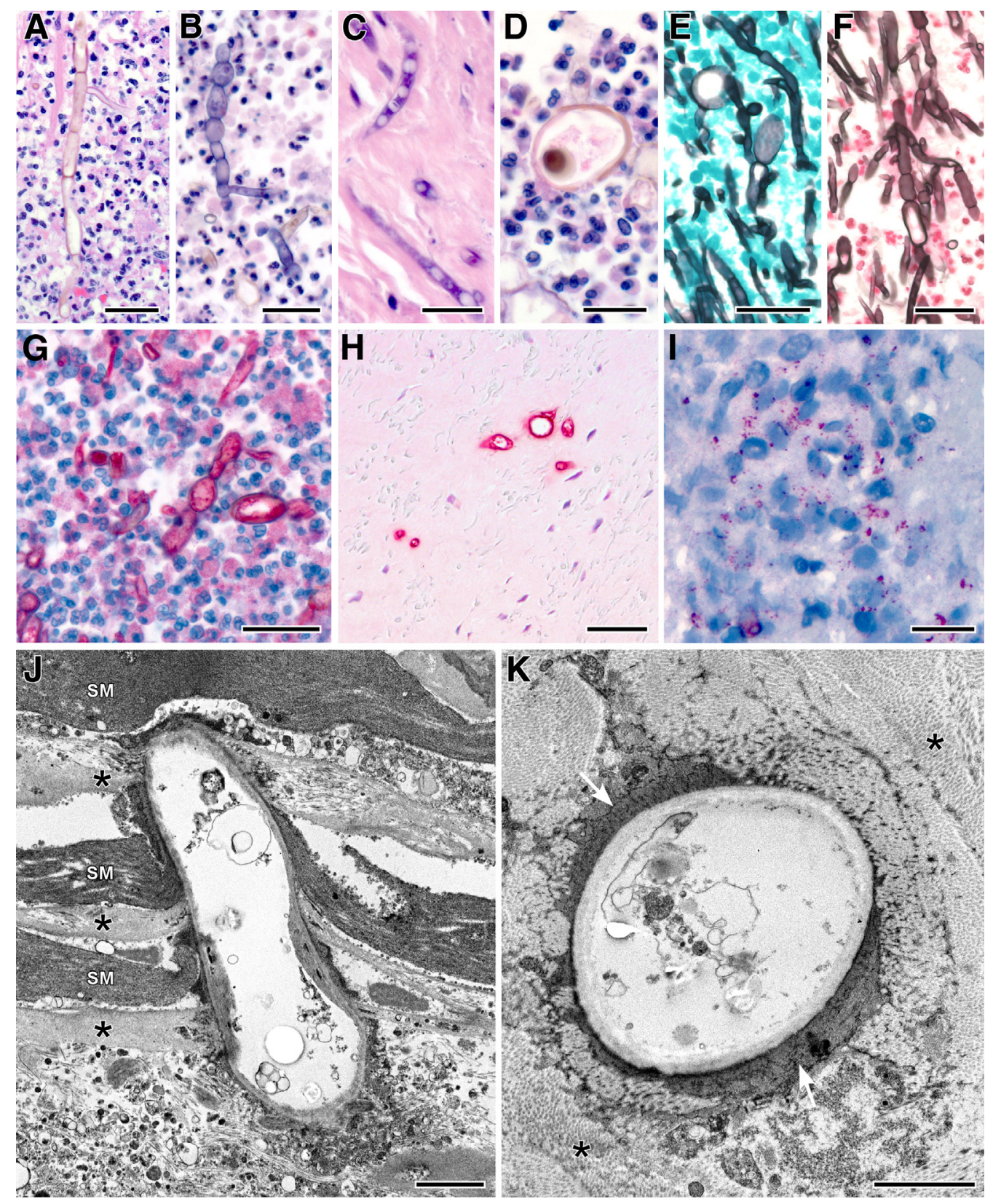

Figure 3 Exserohilum morphological characteristics, detection by IHC, and ultrastructural features of arteritis. A: Pigmented, septate, branching fungal hyphae. B: Chains of yeast-like structures. C: Conidial forms. D: Large spore-like structures. E: GMS stain shows septate fungal hyphae with bulbous dilations. F: FM stain shows septate, branching hyphae of irregular width. G: IHC detection of Exserohilum amid an intense suppurative infiltrate. H: Lack of inflammatory response to Exserohilum in dense avascular connective tissue at paraspinal injection site. I: Detection of granular fungal antigen by IHC in the absence of fungal hyphae. J: Exserohilum penetrating multiple layers of smooth muscle (SM) and collagen (asterisks) in the tunica media of the basilar artery. K: Dissolution of collagen (asterisks) with replacement by electron-dense material (white arrows) surrounding an Exserohilum hypha in the adventitia of a large artery. H\&E staining (A-D); GMS staining (E); FM staining (F); polyfungal immunoalkaline phosphatase staining, naphthol fast red substrate with hematoxylin counterstain $(\mathbf{G}-\mathbf{I})$; and thin-section electron microscopy, $4 \%$ uranyl acetate and lead citrate stain (J and $\mathbf{K})$. Original magnification: $\times 100(\mathbf{A}-\mathbf{C}$ and $\mathbf{E}-\mathbf{I}) ; \times 158$ (D). Scale bars: $30 \mu \mathrm{m}(\mathbf{A}$ and $\mathbf{B}) ; 20 \mu \mathrm{m}(\mathbf{C}-\mathbf{F}$ and $\mathbf{I}) ; 40 \mu \mathrm{m}(\mathbf{G}$ and $\mathbf{H}) ; 2 \mu \mathrm{m}$ ( $\mathbf{J}$ and $\mathbf{K})$.

(score, 3) than epidural/paraspinal or intra-articular injection site infections $(P<0.0001)$. Cases with granulomatous inflammation had a median interval between injection and tissue collection of 48.8 days, whereas those with only necrosuppurative infiltrates had a median interval between injection and tissue collection of only 35.3 days, suggesting a transition from necrosuppurative to granulomatous inflammation over time. There was no statistical significance between detection of fungus by $\mathrm{H} \& \mathrm{E}$ stain and age, sex, interval between injection and tissue collection, interval between initiation of treatment and tissue collection, or detection of Exserohilum in tissues by PCR. However, SI 
Table 3 Detection of Exserohilum in Tissues of 40 Cases Using Various Laboratory Tests

\begin{tabular}{llllll}
\hline \multirow{2}{*}{$\begin{array}{l}\text { Total no. } \\
\text { of cases }\end{array}$} & \multicolumn{6}{l}{ Laboratory test result profile } \\
\cline { 2 - 6 } & H\&E & GMS & IHC & FFPE PCR & Fresh tissue PCR \\
\hline $7^{*}$ & + & + & + & NA & NA \\
$7^{*}$ & - & + & + & - & NA \\
5 & - & + & + & + & NA \\
4 & - & + & + & NA & + \\
$4^{*}$ & - & + & + & NA & - \\
3 & + & + & + & NA & + \\
$3^{*}$ & + & + & + & - & NA \\
2 & + & + & + & + & - \\
1 & + & + & + & + & NA \\
1 & - & - & + & NA & + \\
$1^{*}$ & - & + & + & NA & NA \\
$1^{*}$ & - & - & + & - & - \\
$1^{*}$ & + & + & + & NA & - \\
\hline
\end{tabular}

* Of 24 IHC-positive cases that did not have PCR confirmation on FFPE or fresh tissues, 10 had laboratory confirmation (culture and/or PCR) of Exserohilum from other sample types (CSF or joint fluid).

+ , positive; -, negative; NA, not available (testing not performed).

joint injection sites were more likely than other sites to have detection of predominately granular fungal antigen by IHC $(P=0.0312)$.

\section{Correlation of IHC with PCR}

Fungal PCR was performed on FFPE and/or fresh tissues from 32 of 40 IHC-positive cases. Fungus was detected in $16(50 \%)$ of 32 cases by PCR on one or both tissue types, and no case was PCR positive without also being IHC positive (Table 3). For three cases, PCR was performed on both FFPE and fresh tissues; fresh tissues were negative by PCR for all three cases, whereas FFPE tissues were positive by PCR for two cases. Of 24 cases that were IHC positive but did not have PCR confirmation of Exserohilum from FFPE and/or fresh tissues, 10 (42\%) had other laboratory confirmation (culture and/or PCR) of Exserohilum from other sample types (CSF or joint fluid).

\section{Discussion}

Herein, we characterized the spectrum of histopathological features associated with the largest known series of Exserohilum infections associated with contaminated epidural and intra-articular steroid injections and established IHC as a highly sensitive method for Exserohilum detection in FFPE tissues. Exserohilum species are dematiaceous environmental molds that are known plant pathogens, but rarely infect humans. They are occasionally associated with allergic sinusitis and keratitis in immunocompetent patients and with cutaneous infection after trauma and invasive infection in immunosuppressed, and rarely in immunocompetent, patients. ${ }^{23-26}$ In most cases, invasive infection originates in the upper respiratory tract, with direct infiltration and hematogenous dissemination to other tissues. ${ }^{24}$ Histologically, infections are characterized by necrosis and inflammatory cell infiltrates, which vary from neutrophilic to lymphoplasmacytic to granulomatous; eosinophils are also sometimes prominent. Fungal hyphae are often, but not always, identified within necrotic tissue, and vascular and bone invasion by hyphae are reported. ${ }^{24-29}$ The full spectrum of these lesions was seen among these cases, except for definitive bone invasion or consistently prominent eosinophils (seen in only one case).

The histopathological features of Exserohilum infection seen in this outbreak were similar to those of other more common and well-known fungal agents of human disease. Differentiation of Exserohilum from other fungi in histological sections can be difficult, especially when few hyphae are present for morphological evaluation and when little or no histopathological change accompanies hyphae that are present. PAS and GMS stains aid in identifying most fungi, and FM staining for melanin has traditionally been used to specifically detect dematiaceous fungi; however, some Aspergillus species and few zygomycetes also stain by FM, limiting its usefulness in the diagnosis of phaeohyphomycoses. ${ }^{30}$ Among these cases, polyfungal IHC was the most sensitive method for detecting Exserohilum in FFPE tissues. Although not a specific test for Exserohilum, immunolabeling with this antibody pool, in conjunction with an absence of immunolabeling by IHC using specific anti-Aspergillus species and anti-zygomycete antibodies, was considered compatible with Exserohilum infection in cases from this outbreak. Identification of hyphae was similar for GMS and IHC, but IHC allowed for more efficient screening of specimens because of its superior sensitivity in labeling granular fungal antigen in areas of inflammation that lacked obvious hyphae. This granular antigen was interpreted to be remnants of degraded fungi, which could not be identified specifically as fungal by H\&E or GMS stains. Importantly, IHC was more sensitive for detecting Exserohilum in FFPE tissues than was PCR. The lower sensitivity of PCR may be due, in part, to few intact fungi in tissues and difficulty in breaking down fungal cell walls during the DNA extraction procedure.

The pathophysiological characteristics of fungal meningitis in this outbreak resemble other CNS fungal infections, with fungal angioinvasion and vasculitis resulting in thrombosis and infarction. An apparent predilection of Exserohilum for the basilar structures of the brain was notable and may be the result of spread of organisms through the CSF from the lumbar subarachnoid space to the brain, with pooling of organisms and inflammatory exudates in the basal cisterns and subsequent involvement of the adjacent basilar arteries. It has been shown that radionuclide injection into the lumbar subarachnoid space appears in the basal cisterns within 1 hour and before appearing in other areas of the brain. ${ }^{31}$ Furthermore, a similar predilection for the base of the brain has been seen in cases of Aspergillus meningitis associated 
with epidural spinal anesthesia, ${ }^{7}$ but specific involvement of the base of the brain is not described for CNS Aspergillus infection that occurs hematogenously or by direct invasion from sinuses. The observation of abundant fungi in the adventitial and perivascular tissues, but relatively few fungi in arterial lumens, suggests migration of fungus into, rather than out of, vessels at this location. This further supports the hypothesis of Exserohilum migration from lumbar spine to brain through the CSF, with subsequent vascular invasion, rather than migration through the vasculature.

There may also be an as yet unexplained tropism of the fungus for this area related to vascular structural, physiological, or hemodynamic factors. Animal studies have shown a direct communication between the adventitia of cerebral arteries and surrounding CSF. ${ }^{32}$ If such a communication exists in humans, it could facilitate fungal angioinvasion in the major CNS arteries. The organism's metabolic requirements for oxygen and iron concentrated in arterial erythrocytes may be an impetus, and protease digestion or physical disruption of the vessel wall may provide the means, for angioinvasion, as described for other fungi. ${ }^{33-37}$ Pectinase production by $E$. rostratum has been demonstrated and likely plays a role in its phytopathogenicity ${ }^{38}$; by analogy, production of proteases may result in digestion and hydrolysis of structural proteins in and around the arterial wall, as suggested by our electron microscopic findings. In the absence of accidental dural puncture during steroid injection, penetration of connective tissue would allow the fungus to initially invade the dura to gain access to the CSF after epidural inoculation. Although the dura is typically regarded as impervious and contiguous with the epineurium of spinal nerves, there is evidence that normal CSF resorption involves some flow across the dura and cranial and spinal nerve sheaths, with uptake by lymphatics. ${ }^{32,39,40}$ This implies some degree of dural permeability, which may be another potential means of initial fungal entry into the CSF.

Direct extension of fungi or inflammation from the meninges into the subjacent neural parenchyma was only observed in a few cases near the site of spinal epidural injection, but was not observed in brain tissues. This is in contrast to other common fungal infections, which readily invade neural tissues by direct extension. ${ }^{41}$ For seven patients with fatal meningitis, extra-CNS tissues were also submitted for evaluation for disseminated infection. Neither fungal organisms nor pathology attributed to fungal infection were identified in any non-CNS tissue for any case. These findings suggest a lack of systemic dissemination of Exserohilum, which was surprising given the extensive meningeal vascular invasion, high fungal burdens, and the occurrence of systemic involvement with other angioinvasive fungal infections, especially in immunocompromised patients. ${ }^{42,43}$ Fungal inoculation directly into the epidural space with rapid development of meningitis and death may account for the lack of systemic dissemination observed in these cases.

In general, a type 1 helper T-cell-mediated immune response to some fungal infections leads to phagocytic destruction of fungal elements and clearance of infection. ${ }^{44}$ Polymorphonuclear cells and macrophages predominate at sites of infection, often with granulomatous inflammation associated with fungal clearance. In this case series, those with granulomatous inflammation had an interval between steroid injection and tissue collection of approximately 2 weeks longer than cases with only necrosuppurative inflammation, suggesting a time-dependent transition from necrosuppurative to granulomatous infiltrates, which could be consistent with such a type 1 helper T-cell-mediated immune response. Corticosteroids have an overall suppressive effect on cellmediated immunity by reducing phagocytic activity and suppressing cytokine production. All patients in this outbreak had at least one steroid injection concomitantly with fungal inoculation, and many likely had multiple prior steroid injections or other comorbidities, which may have contributed to an impaired immune response to Exserohilum. Although the meningeal inflammation associated with fungal elements was often florid, an intriguing finding was the lack of inflammation in some tissues containing numerous hyphae, particularly the tunica media of large arteries and dense connective tissues at injection sites. Although it is tempting to attribute this lack of inflammation to the immunosuppressive effects of steroids, studies of other fungal infections in systemically immunocompromised patients would contradict this theory. In Aspergillus infections, patients immunosuppressed with glucocorticoids have a more fulminant neutrophilic inflammatory infiltrate, with lower fungal burdens and less angioinvasion than chemotherapeutically treated neutropenic patients. ${ }^{45}$ Other as yet undefined mechanisms, such as production of fungal secondary metabolites, may play a role in immune evasion by Exserohilum. Further studies are needed to better characterize the pathogenesis of Exserohilum infection and to investigate the potential effects of various underlying host factors, including immunosuppression from steroid administration or other comorbidities.

Tissues included in this evaluation were received at a reference diagnostic pathology laboratory (Infectious Diseases Pathology Branch, CDC) from multiple medical facilities across seven US states and encompassed a variety of injection locations and clinical presentations. Although this allowed for a cross-sectional analysis of the affected population in this outbreak, associated limitations included incomplete reporting of clinical data and gross pathological findings, lack of complete tissue sets for evaluation, and submission of samples that were not representative of the reported pathological findings. Despite these limitations, we were able to confirm fungal infection in FFPE tissues by IHC in these 40 cases, and to gain insight into the pathogenesis of Exserohilum meningitis after iatrogenic epidural inoculation.

In summary, E. rostratum infections associated with epidural injections had consistent histopathological features of meningitis and vasculitis with thrombosis and fungal angioinvasion, with prominent involvement of the basilar arterial circulation. The range of cellular inflammatory 
patterns and fungal burden in tissues varied and was likely influenced by clinical factors, such as amount of fungus in the inoculum, interval between injection and tissue collection, administration of antifungal medications, and individual host factors (eg, underlying comorbidities and individual host genetic variability). The concurrent inoculation of steroids and fungus likely also influenced the pathophysiology of infections and may have contributed to morbidity and mortality in this outbreak. Special stains and IHC were useful for identifying fungus in FFPE tissues, and IHC was more sensitive than even molecular methods for fungal detection in FFPE samples. Little is known about Exserohilum as a human pathogen, and additional studies are warranted to further investigate both intrinsic properties of the fungus and human factors that contribute to infection and disease pathogenesis.

\section{Acknowledgments}

We thank the following public health partners in state and local health departments, public health laboratories, and hospital and medical examiner offices: Carol Rauch, Karen Cline-Parhamovich, Amy Tharp, Li Chen, Joseph Prahlow, Deborah Radisch, Lauren Scott, Ann Schmitz, Tom Torok, Carina Blackmore, Timothy Whitman, Peter Jelsma, Paula Gibbs, Marie-Claire Rowlinson, Manuel Leal, and Dorothy Baynham.

\section{References}

1. Carrino JA, Morrison WB, Parker L, Schweitzer ME, Levin DC, Sunshine JH: Spinal injection procedures: volume, provider distribution, and reimbursement in the U.S. medicare population from 1993 to 1999. Radiology 2002, 225:723-729

2. Windsor RE, Storm S, Sugar R: Prevention and management of complications resulting from common spinal injections. Pain Physician 2003, 6:473-483

3. Derby R, Lee SH, Kim BJ, Chen Y, Seo KS: Complications following cervical epidural steroid injections by expert interventionalists in 2003. Pain Physician 2004, 7:445-449

4. Kabbara A, Rosenberg SK, Untal C: Methicillin-resistant Staphylococcus aureus epidural abscess after transforaminal epidural steroid injection. Pain Physician 2004, 7:269-272

5. Gaul C, Neundörfer B, Winterholler M: Iatrogenic (para-) spinal abscesses and meningitis following injection therapy for low back pain. Pain 2005, 116:407-410

6. Simopoulos TT, Kraemer JJ, Glazer P, Bajwa ZH: Vertebral osteomyelitis: a potentially catastrophic outcome after lumbar epidural steroid injection. Pain Physician 2008, 11:693-697

7. Rodrigo N, Perera KN, Ranwala R, Jayasinghe S, Warnakulasuriya A, Hapuarachchi S: Aspergillus meningitis following spinal anaesthesia for caesarean section in Colombo. Sri Lanka Int J Obstet Anesth 2007, $16: 256-260$

8. Saigal G, Donovan Post MJ, Kozic D: Thoracic intradural Aspergillus abscess formation following epidural steroid injection. AJNR Am J Neuroradiol 2004, 25:642-644

9. Kolbe AB, McKinney AM, Kendi AT, Misselt D: Aspergillus meningitis and discitis from low-back procedures in an immunocompetent patient. Acta Radiol 2007, 48:687-689
10. Civen R, Vugia DJ, Alexander R, Brunner W, Taylor S, Parris N, Wasserman R, Abbott S, Werner SB, Rosenberg J: Outbreak of Serratia marcescens infections following injection of betamethasone compounded at a community pharmacy. Clin Infect Dis 2006, 43: 831-837

11. Nakashima AK, McCarthy MA, Martone WJ, Anderson RL: Epidemic septic arthritis caused by Serratia marcescens and associated with a benzalkonium chloride antiseptic. J Clin Microbiol 1987, 25: 1014-1018

12. Cohen AL, Ridpath A, Noble-Wang J, Jensen B, Peterson AM, Arduino M, Jernigan D, Srinivasan A: Outbreak of Serratia marcescens bloodstream and central nervous system infections after interventional pain management procedures. Clin J Pain 2008, 24:374-380

13. Wong MR, Del Rosso P, Heine L, Volpe V, Lee L, Kornblum J, Lin Y, Layton M, Weiss D: An outbreak of Klebsiella pneumoniae and Enterobacter aerogenes bacteremia after interventional pain management procedures, New York City, 2008. Reg Anesth Pain Med 2010, 35:496-499

14. Centers for Disease Control and Prevention (CDC): Exophiala infection from contaminated injectable steroids prepared by a compounding pharmacy-United States, July-November 2002. MMWR Morb Mortal Wkly Rep 2002, 51:1109-1112

15. Centers for Disease Control and Prevention (CDC): Multistate outbreak of fungal infection associated with injection of methylprednisolone acetate solution from a single compounding pharmacy: United States, 2012. MMWR Morb Mortal Wkly Rep 2012, 61:839-842

16. Pettit AC, Kropski JA, Castilho JL, Schmitz JE, Rauch CA, Mobley BC, Wang XJ, Spires SS, Pugh ME: The index case for the fungal meningitis outbreak in the United States. N Engl J Med 2012, 367:2119-2125

17. Smith RM, Schaefer MK, Kainer MA, Wise M, Finks J, Duwve J, Fontaine E, Chu A, Carothers B, Reilly A, Fiedler J, Wiese AD, Feaster C, Gibson L, Griese S, Purfield A, Cleveland AA, Benedict K, Harris JR, Brandt ME, Blau D, Jernigan J, Weber JT, Park BJ, Multistate Fungal Infection Outbreak Response Team: Fungal infections associated with contaminated methylprednisolone injections: preliminary report. N Engl J Med 2012, [Epub ahead of print]

18. Bell WR, Dalton JB, McCall CM, Karram S, Pearce DT, Memon W, Lee R, Carroll KC, Lyons JL, Gireesh ED, Trivedi JB, Cettomai D, Smith BR, Chang T, Tochen L, Ratchford JN, Harrison DM, Ostrow LW, Stevens RD, Chen L, Zhang SX: Iatrogenic Exserohilum infection of the central nervous system: mycological identification and histopathological findings. Mod Pathol 2013, 26:166-170

19. Hayat MA: Principles and Techniques of Electron Microscopy, ed 3. Boca Raton, CRC Press, 1989, pp 134-136

20. Mollenhauer HH: Plastic embedding mixtures for use in electron microscopy. Stain Technol 1964, 39:111-114

21. Gade L, Scheel C, Pham CD, Lindsley MD, Iqbal N, Cleveland AA, Whitney AM, Lockhart SR, Brandt ME, Litvintseva AP: Detection of fungal DNA in human body fluids and tissues during a multistate outbreak of fungal meningitis and other infections. Eukaryot Cell 2013, 12:677-683

22. Muñoz-Cadavid C, Rudd S, Zaki SR, Patel M, Moser SA, Brandt ME, Gómez BL: Improving molecular detection of fungal DNA in formalin-fixed paraffin-embedded tissues: comparison of five tissue DNA extraction methods using panfungal PCR. J Clin Microbiol 2010, 48:2147-2153

23. Juhas E, Reyes-Mugica M, Michaels MG, Grunwaldt LJ, Gehris RP: Exserohilum infection in an immunocompromised neonate. Pediatr Dermatol 2012. http://dx.doi.org/10.1111/j.1525-1470.2012.01829.x

24. Adler A, Yaniv I, Samra Z, Yacobovich J, Fisher S, Avrahami G, Levy I: Exserohilum: an emerging human pathogen. Eur J Clin Microbiol Infect Dis 2006, 25:247-253

25. Adam RD, Paquin ML, Petersen EA, Saubolle MA, Rinaldi MG, Corcoran JG, Galgiani JN, Sobonya RE: Phaeohyphomycosis caused by the fungal genera Bipolaris and Exserohilum: a report of 9 cases and review of the literature. Medicine (Baltimore) 1986, 65:203-217 
26. Padhye AA, Ajello L, Wieden MA, Steinbronn KK: Phaeohyphomycosis of the nasal sinuses caused by a new species of Exserohilum. $\mathbf{J}$ Clin Microbiol 1986, 24:245-249

27. Lasala PR, Smith MB, McGinnis MR, Sackey K, Patel JA, Qiu S: Invasive Exserohilum sinusitis in a patient with aplastic anemia. Pediatr Infect Dis J 2005, 24:939-941

28. Aquino VM, Norvell JM, Krisher K, Mustafa MM: Fatal disseminated infection due to Exserohilum rostratum in a patient with aplastic anemia: case report and review. Clin Infect Dis 1995, 20:176-178

29. McGinnis MR, Rinaldi MG, Winn RE: Emerging agents of phaeohyphomycosis: pathogenic species of Bipolaris and Exserohilum. J Clin Microbiol 1986, 24:250-259

30. Kimura M, McGinnis MR: Fontana-Masson-stained tissue from culture-proven mycoses. Arch Pathol Lab Med 1998, 122:1107-1111

31. James AE Jr, DeLand FH, Hodges FJ 3rd, Wagner HN Jr: Cerebrospinal fluid (CSF) scanning: cisternography. Am J Roentgenol Radium Ther Nucl Med 1970, 110:74-87

32. Sakka L, Coll G, Chazal J: Anatomy and physiology of cerebrospinal fluid. Eur Ann Otorhinolaryngol Head Neck Dis 2011, 128:309-316

33. Denning DW, Ward PN, Fenelon LE, Benbow EW: Lack of vessel wall elastolysis in human invasive pulmonary aspergillosis. Infect Immun 1992, 60:5153-5156

34. Chung D, Haas H, Cramer RA: Coordination of hypoxia adaptation and iron homeostasis in human pathogenic fungi. Front Microbiol 2012, 3:381

35. Ibrahim AS: Host cell invasion in mucormycosis: role of iron. Curr Opin Microbiol 2011, 14:406-411
36. Ben-Ami R, Lewis RE, Kontoyiannis DP: Enemy of the (immunosuppressed) state: an update on the pathogenesis of Aspergillus fumigatus infection. Br J Haematol 2010, 150:406-417

37. Norlinah MI, Ngow HA, Hamidon BB: Angioinvasive cerebral aspergillosis presenting as acute ischaemic stroke in a patient with diabetes mellitus. Singapore Med J 2007, 48:e1-e4

38. Ortega J: Production of pectinases by Exserohilum rostratum. Texas $\mathbf{J}$ Sci 1994, 46:79-86

39. Koh L, Zakharov A, Johnston M: Integration of the subarachnoid space and lymphatics: is it time to embrace a new concept of cerebrospinal fluid absorption? Cerebrospinal Fluid Res 2005, 2:6

40. Edsbagge M, Tisell M, Jacobsson L, Wikkelso C: Spinal CSF absorption in healthy individuals. Am J Physiol Regul Integr Comp Physiol 2004, 287:R1450-R1455

41. Ellison D, Love S, Chimelli L, Harding B, Lowe J, Roberts GW, Vinters HW. Edited by Hodgson S, (Ed). Fungal infections. Neuropathology. Barcelona, Mosby International Ltd, 1998, pp 17.2-17.6

42. Lopes Bezerra LM, Filler SG: Interactions of Aspergillus fumigatus with endothelial cells: internalization, injury, and stimulation of tissue factor activity. Blood 2004, 103:2143-2149

43. Revankar SG, Patterson JE, Sutton DA, Pullen R, Rinaldi MG: Disseminated phaeohyphomycosis: review of an emerging mycosis. Clin Infect Dis 2002, 34:467-476

44. Blanco JL, Garcia ME: Immune response to fungal infections. Vet Immunol Immunopathol 2008, 125:47-70

45. Lewis RE, Kontoyiannis DP: Invasive aspergillosis in glucocorticoidtreated patients. Med Mycol 2009, 47(Suppl 1):S271-S281 